\title{
A Refined Technique for Management of Nasal Flaring: The Quest for the Holy Grail of Alar Base Modification
}

\author{
Kapil S Agrawal, Mansi Pabari, Raghav Shrotriya \\ Department of Plastic Surgery, KEM Hospital, Mumbai, India
}

\begin{abstract}
"A smile is happiness you'll find right under your nose"- Tom Wilson. This quote holds true just for patients, not for surgeons. The correction of the nose always poses a challenge to the cosmetic surgeon. Deformities of the external and internal nose may be congenital or acquired and may be secondary to soft tissue and/or osseo-cartilaginous abnormalities, leading to aesthetic and/or functional consequences. Alar flare poses a common problem, sometimes alone and sometimes in conjunction with other external deformities. Alar base reduction is generally considered when the interalar distance exceeds the intercanthal distance. It has been well documented that this simple additional procedure brings about a substantial enhancement in the nose. Various techniques have been described and used in the past, each having their benefits and drawbacks, with the modified Weir wedge excision, Aufricht nasal sill excision, and Bernstein $\mathrm{V}-\mathrm{Y}$ advancement being the common ones. We hereby describe a technique that is simple yet effective in achieving the desired results and at the same time aims at preventing relapse to obtain satisfactory long term results.
\end{abstract}

Keywords Ala / Alar / Alar fold / Rhinoplasty
Correspondence: Raghav Shrotriya Department of Plastic Surgery, Gynec. Wing, 2nd floor, KEM Hospital, Parel, Mumbai 400012, India

Tel: +91-22-2410-7218

Fax: +91-22-2401-7518

E-mail:dr.raghav.s@gmail.com
No potential conflict of interest relevant to this article was reported.

\section{INTRODUCTION}

Deformities of the nasal alar base are a common finding in secondary rhinoplasty. It may be a primary deformity which was not addressed initially or might have developed as a secondary deformity, for instance, excessive alar flaring developed after reduction of tip projection. A minor change in the alar base can enhance the overall effect of a nose job, yet recent studies reveal a hesitation towards the use of such techniques during primary rhinoplasties due to more extensive alar base narrowing during revision procedures $[1,2]$.

We, herein describe a modified technique which preserves the alar groove and can also be used in conjunction with open rhi- noplasty procedures to bring about a significant improvement in the alar flare. The key is to follow the curvatures of the incision well along the existing curves of the ala and create a feeding notch so as to produce long-lasting results.

\section{IDEA}

Marking is done as shown in Figs. 1A, B, 2, and 3. Measurement should be performed precisely using calipers. What is left behind after the excision should be the guiding principle for the amount of tissue to be excised even though the excised specimen may be asymmetric.

After infiltration, the skin is excised from the marked area. Though 


\section{Fig. 1. Schematic diagram of the author's technique}

(A) Marking for skin excision on lateral view. Point ' $x$ ' is the $V$-flap of alar margin and point ' $y$ ' is the inverted ' $v$ ' notch created in the sill after combined excision of the ala and sill skin. (B) Markings as seen on basal view. Flap ' $x$ ' will be fed into notch ' $y$ '. (C) Half-buried 6-0 monofilament suture to feed alar V-flap into the notch formed by sill excision. (D) Raw area shown as dotted pink. (E) Final suture line.

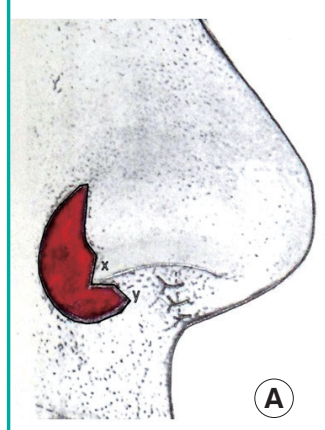

(A)

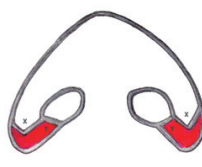

(B)

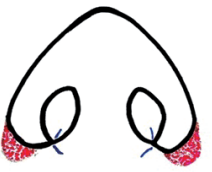

(D)

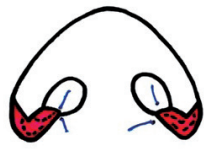

(C)

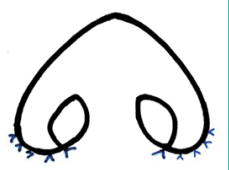

(E)

\section{Fig. 2. Marking on basal view}

Showing 'V' alar margin flap and marking for sill reduction.

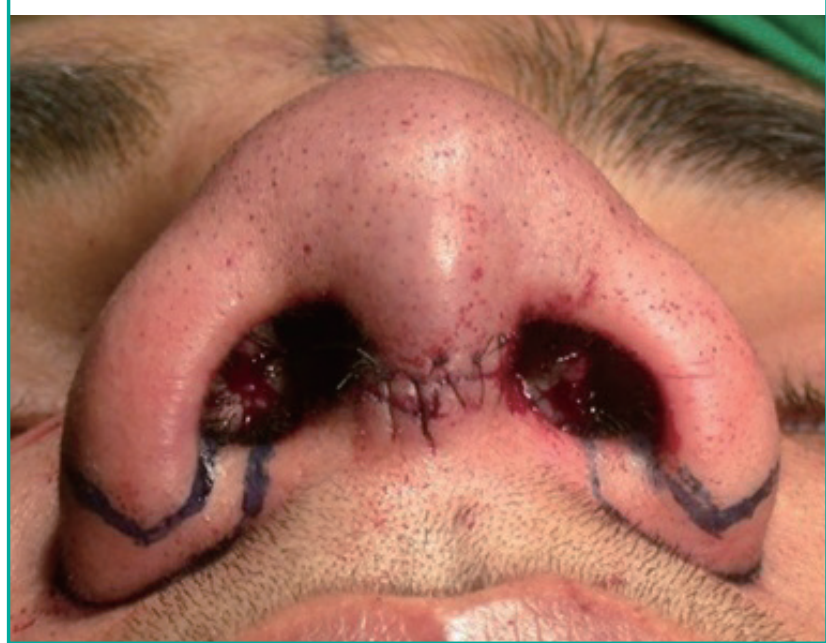

few authors recommend making the lower incision 1-2 mm above the alar groove crease, this author (K.S.A.) performs the lower incision in the alar groove itself. Any bleeding point is cauterized. The ' $\mathrm{V}$ ' alar margin flap moves in and sits well in the slot formed by the excision of the sill and is sutured by half-buried mattress sutures with 6-0 polypropylene (Figs. 1C, D, 4, and 5). A few absorbable sutures may be used to reduce the tension in the alar groove region, and then the skin margins are approximated using 6-0 polypropylene. After cleaning the suture line (Fig. 1E), antibiotic ointment is applied. In the postoperative period, cleaning of the suture line is done regularly to avoid maceration. The suture removal is carried out on the fifth postoperative day. The patient is advised not to touch or massage the suture line to avoid widening of the scar.

\section{Fig. 3. Marking on lateral view}

A patient who underwent surgery for alar base modification: showing concavo-convex skin-only excision on lateral view.

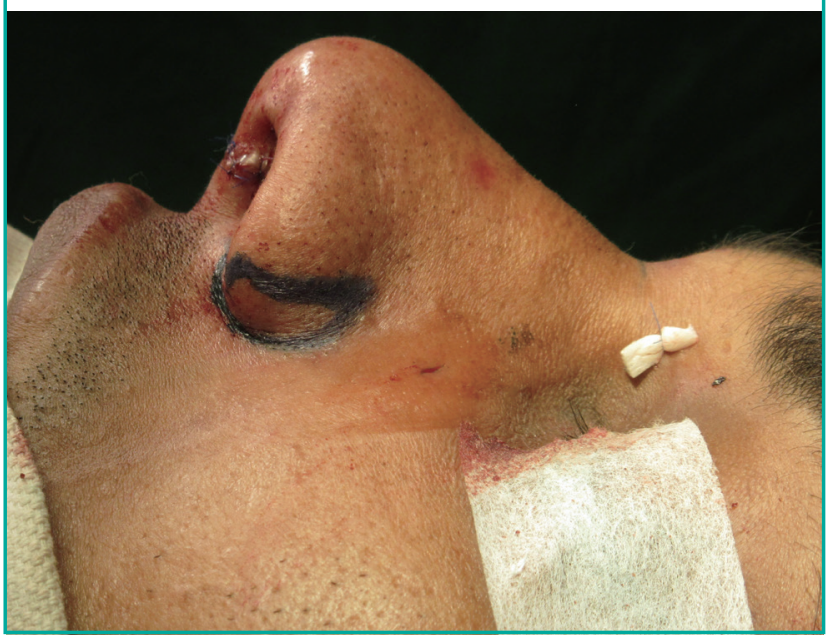

Fig. 4. Final suture line on basal view

Note that the ' $V$ ' alar margin flap moves in and sits well in the slot formed by excision of the sill.

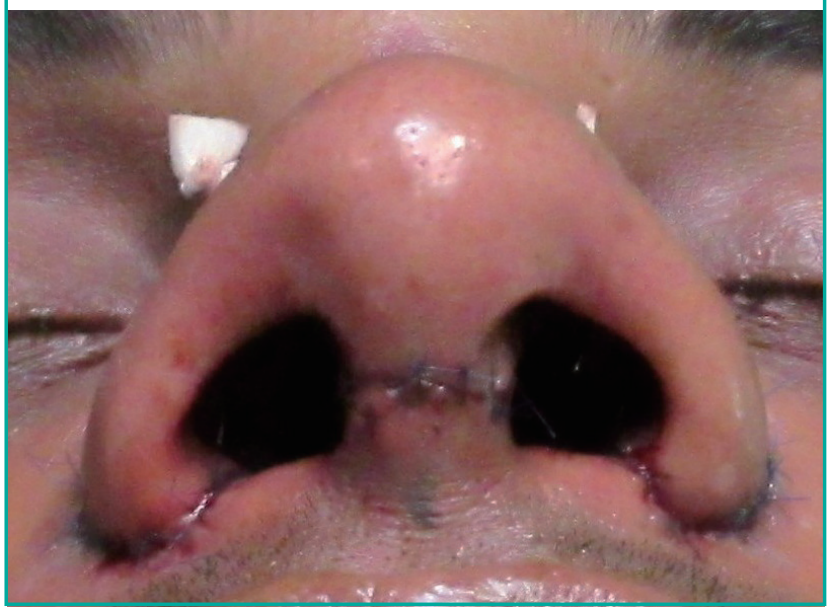

\section{DISCUSSION}

Alar flare poses a common problem, sometimes alone and sometimes in conjunction with other external deformities. Alar base reduction is generally considered when the interalar distance exceeds the intercanthal distance. The history of alar base reduction reveals a great variety of surgical maneuvers.

Robert Weir first reported the external alar wedge excision in 1892 to correct alar flaring, hiding the incision in the alar-facial groove [3]. Joseph and Milstein modified the technique in 1931 to avoid external scarring by including internal excisions from the nostril base and vestibular floor [4]. Various modifications have been attempted since then to achieve acceptable results. Foda [5] described the 'boomerang excision technique', which 

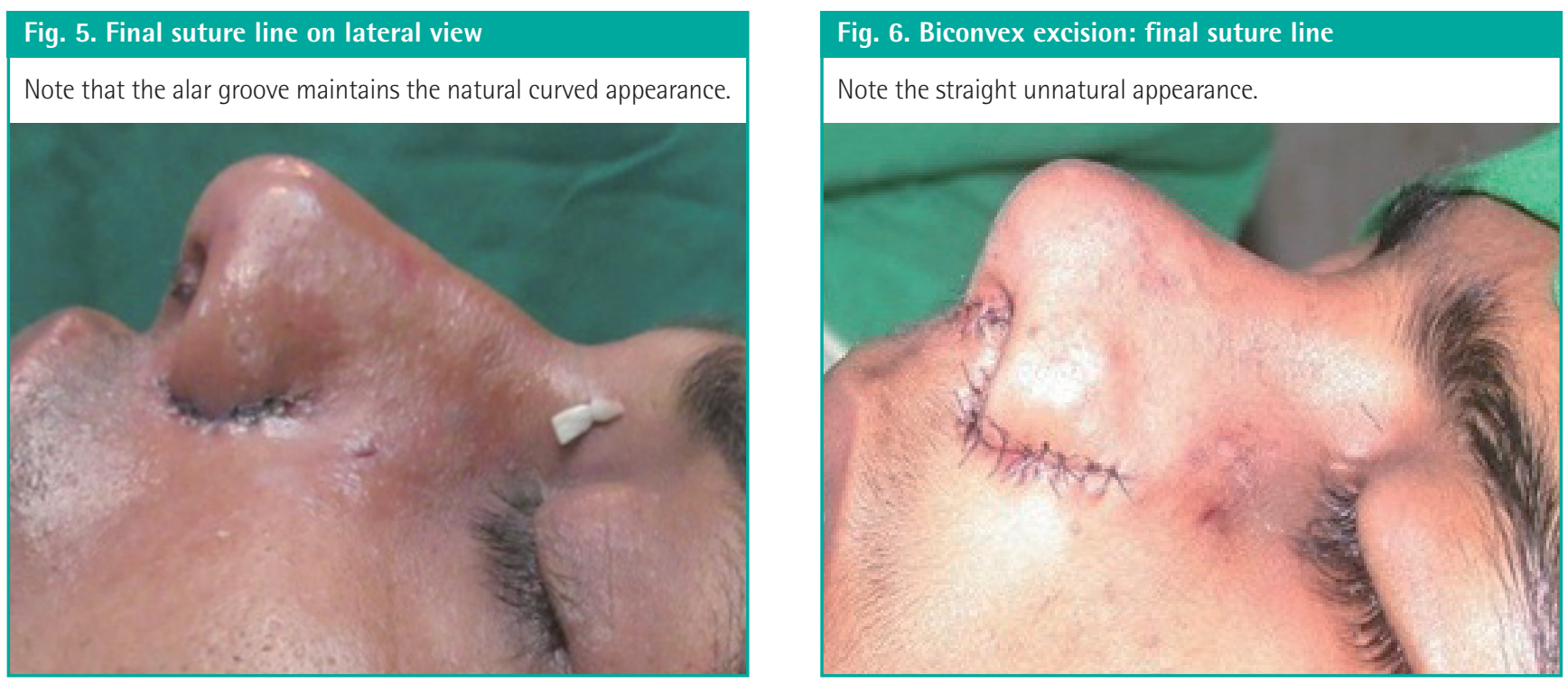

\section{Fig. 7. Clinical photograph}

(A) A patient who underwent surgery for alar base modification. Preoperative photograph. (B) One year postoperative photograph. Note that the $\mathrm{V}$ flap has moved easily, producing a natural appearance on basal view, maintaining the normal alar groove.
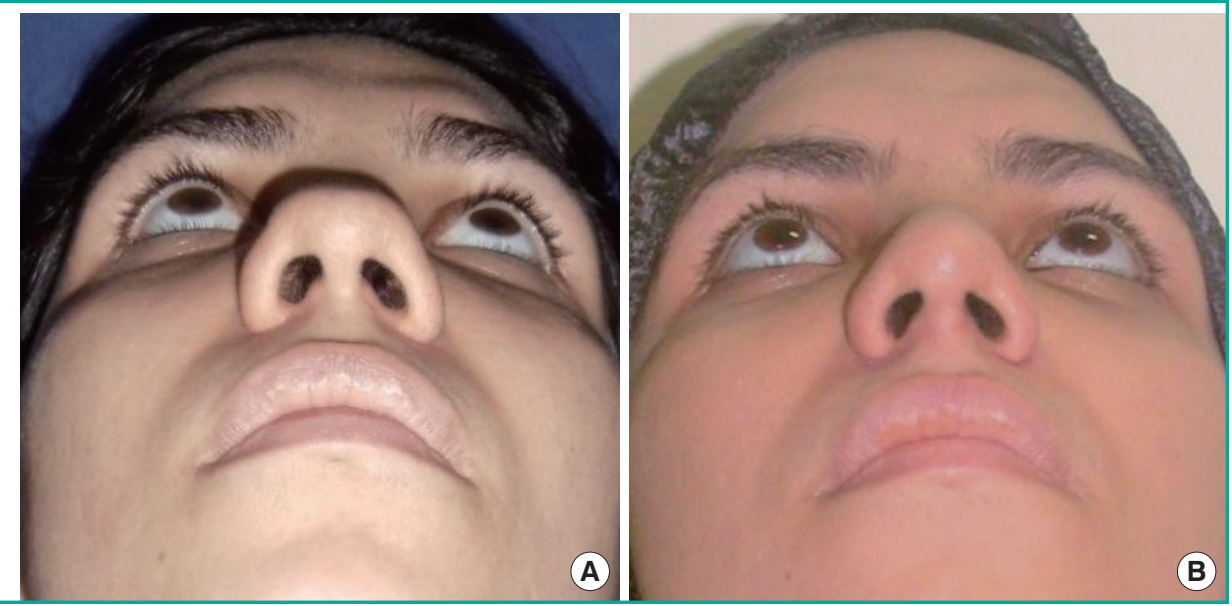

combines external alar wedge resection with internal vestibular floor excision. The internal excision was inclined $30^{\circ}-45^{\circ}$ laterally to form the inner limb of the boomerang.

Kridel and Castellano [6] used a combination of three different techniques on 124 patients. He established a set of indications for each type of alar base flare and selected the necessary technique. He used the wedge excision technique for a wide nasal base on frontal view. It spares the nostril and hence prevents violation of the curved internal nostril border. Aufricht [7] used nasal sill excision in cases with an enlarged nasal sill width. Bernstein's [8] V-Y advancement was used in cases where the lateral insertion of the ala was responsible for the flared look in the presence of an already narrow nasal sill.

Improper correction of alar base deformities may lead to aesthetic as well as functional complications in the form of unsightly scars, short and straight ala, narrowing and/or stenosis of the nostrils, and asymmetry of the nostrils and alar bases. The key to avoiding complications is that this maneuver should not be undertaken without a strong indication, it should be planned preoperatively, must be executed at the end of the rhinoplasty if need be, and the surgeon should excise conservatively. Various indications are alar flaring, increased inter-alar distance, wide nostril sill, and asymmetries of the ala (to be recorded preoperatively on basal view photographs).

Sheen modified alar base excision and classified techniques on the basis of their objectives [9]: Type 1, alar wedge excision; Type 2, combined alar base and nostril sill excision; Type 3, sill excision alone; Type 4, combined alar base, nostril sill and nostril floor excision.

The senior author (K.S.A.) has introduced a few modifications to optimize the outcome, keeping in mind the occasional need to reverse the procedure. The author never excises a biconvex (elliptical) wedge, as it makes the alar base concave, and when this concavity meets the concavity of the alar facial groove, it be- 
comes a straight line and looks unnatural (Fig. 6). The normal curvature is followed and a concavo-convex skin wedge is excised (Fig. 2). In cases where a combined alar base and sill excision is required, the author takes a V-flap on the alar rim. This Vflap, when moved medially, maintains the normal alar curve, deepens the alar facial groove where the scar is hidden, and avoids contraction of the resultant scar over the sill. It also serves to avoid a 'Q' deformity of the nostril, which is a telltale sign of alar base modification.

The authors avoid full-thickness excision of the ala. Only the skin is excised from the marked area. Advantages of this modification are that it does not penetrate the vestibular skin and preserves the blood supply, which may be a concern in open rhinoplasty. It also prevents scarring and chances of nostril stenosis and saves a great deal of time and energy in suturing on the inner side of the nose. In the worst case scenario, if the procedure needs to be reversed partially or completely (due to improper planning or execution), the excised skin can be used as a fullthickness skin graft. The author has used this technique in more than 25 cases, producing excellent outcomes for management of alar flaring. A representative outcome is shown in Fig. 7.

An important point to remember here is that the patient should never be advised to massage the alar crease scar. Overzealous massage in this region causes stretching of the scar, flattening or loss of the alar groove, and a visible scar.

Time and again, various maneuvers have been attempted with varying success in order to reduce the alar flare and hence provide an aesthetically pleasing appearance to the nose. The technique that we have described is simple and efficient for achieving acceptable results in one of the trickiest steps of rhinoplasty.

\section{ORCID}

Raghav Shrotriya http://orcid.org/0000-0002-1604-0520

\section{REFERENCES}

1. Daniel RK. Middle Eastern rhinoplasty in the United States: Part II. Secondary rhinoplasty. Plast Reconstr Surg 2009; 124:1640-8.

2. Saltman BE, Pearlman SJ. Incidence of alarplasty in primary and revision rhinoplasty in a private practice setting. Arch Facial Plast Surg 2009;11:114-8.

3. Weir RF. On restoring noses without scarring the face. N Y Med J 1892;56:449-54.

4. Anderson JR. A reasoned approach to nasal base surgery. Arch Otolaryngol 1984;110:349-58.

5. Foda HM. Nasal base reduction. J Asthet Chir 2014;7:8-15.

6. Kridel RH, Castellano RD. A simplified approach to alar base reduction: a review of 124 patients over 20 years. Arch Facial Plast Surg 2005; 7:81-93.

7. Aufricht G. A few hints and surgical details in rhinoplasty. Laryngoscope 1943;53:317-35.

8. Bernstein L. Esthetic anatomy of the nose. Laryngoscope 1972;82:1323-30.

9. Daniel RK, Regnault P. Rhinoplasty: aesthetic plastic surgery. Boston: Little, Brown; 1993. 